ARTÍ́CULO DE REVISIÓN

\title{
Pancreatitis poscolangiopancreatografía retrógrada endoscópica
}

\author{
Mariana Quintanar-Martínez y Félix I. Téllez-Ávila* \\ Departamento de Endoscopia Gastrointestinal, Instituto Nacional de Ciencias Médicas y Nutrición Salvador Zubirán, Ciudad de México, México
}

\begin{abstract}
Resumen
Una adecuada selección del paciente, la estratificación del riesgo preprocedimiento, modificaciones a la técnica de la colangiopancreatografía retrógrada endoscópica (CPRE) y la profilaxis farmacológica son estrategias que han demostrado ser costo-efectivas en la prevención de pancreatitis post-CPRE. Esta revisión se centrará en los factores de riesgo asociados a pancreatitis post-CPRE y en las intervenciones que han demostrado mayor beneficio para disminuir esta complicación.
\end{abstract}

Palabras clave: Pancreatitis. CPRE. Pancreatitis post-CPRE.

\section{Post endoscopic retrograde cholangiopancreatography pancreatitis}

\begin{abstract}
Appropriate patient selection, pre-procedure risk stratification, modifications to the endoscopic retrograde cholangiopancreatography (ERCP) technique, and pharmacoprevention are cost-effective strategies to prevent post-ERCP pancreatitis. This review will focus on the risk factors associated with post-ERCP pancreatitis and the prophylactic interventions that have proven to be effective in reducing this complication.
\end{abstract}

Key words: Pancreatitis. ERCP. Post-ERCP pancreatitis.

\section{Introducción}

La colangiopancreatografía retrógrada endoscópica (CPRE) es un herramienta útil en el diagnóstico y tratamiento de diversas patologías pancreatobiliares (Tabla 1) $)^{1,2}$. En los últimos años se ha incrementado de forma significativa el número de procedimientos realizados con fines terapéuticos y actualmente en el mundo se efectúan > 1.3 millones de estudios por año $0^{3,4}$.

La tasa global de complicaciones post-CPRE reportada en la literatura es del $4-20.5 \% \%^{5-14}$. Esta variabilidad tan amplia se debe a las características de los pacientes incluidos en los diferentes estudios, al volumen y a la complejidad de los procedimientos efectuados en cada centro, a la experiencia del endoscopista y en los últimos años a la implementación de medidas profilácticas encaminadas a reducir los eventos de pancreatitis.

La pancreatitis post-CPRE (PPC) es el evento adverso más frecuente y al mismo tiempo el más temido, por la morbimortalidad asociada y el impacto económico derivado de su atención médica. De acuerdo con una estimación realizada en EE.UU., se calcula que los costos generados por esta complicación oscilan alrededor de los 200 millones de dólares por año ${ }^{15}$.
Correspondencia:

*Félix I. Téllez-Ávila

E-mail: felixtelleza@gmail.com

DOl: 10.24875/END.M20000202
Disponible en internet: 10-09-2020

Endoscopia. 2020;32(3):97-107

www.endoscopia-ameg.com 
Tabla 1. Principales indicaciones de CPRE

\section{Colangiografía}

Ictericia con sospecha de obstrucción biliar

Coledocolitiasis (riesgo alto)

Estenosis benigna y maligna de la vía biliar

Colangitis

Fuga o fístula biliar

Estenosis papilar

Facilitar la colangioscopia

Sospecha de disfunción del esfínter de Oddi tipo 1 > tipo 2

Alta sospecha de colangitis esclerosante primaria temprana (con estudios no invasivos negativos)

Tratamiento de hemobilia

Pancreatografía

Sospecha de fuga o fístula pancreática

Tratamiento de estenosis pancreática sintomática

Tratamiento de litiasis pancreática intraductal sintomática

Drenaje transpapilar de pseudoquistes pancreáticos

Facilitar la pancreatoscopia

Identificar el conducto pancreático previo a una ampulectomía

CPRE: colangiopancreatografía retrógrada endoscópica. Adaptada de Solomon, et al., $2019^{1}$ y Easler, et al., $2019^{2}$.

\section{Epidemiología}

En dos revisiones sistemáticas se encontró que la incidencia de PPC es del 3.4-9.7\%, siendo mayor en los pacientes con factores de alto riesgo (14.7-30\%) y en aquellos procedimientos ejecutados por endoscopistas no expertos $(12 \%)^{9,14,15}$. La mayor parte de las PPC son leves y la mortalidad relacionada es baja $(0.1-0.7 \%)^{15}$. No obstante, el $5-12.6 \%$ de los casos tendrán criterios de gravedad, con una mortalidad incrementada que fluctúa entre el 3 y el $30 \%^{9,15,16}$.

\section{Diagnóstico}

El diagnóstico se puede establecer con cualquiera de las siguientes definiciones:

- Criterios del consenso de Cotton (1991). Aparición o incremento de dolor abdominal pancreático, elevación de enzimas pancreáticas $\geq 3$ veces el límite superior normal 24 horas posteriores al procedimiento y la necesidad de hospitalización ( $\geq 2$ días). La gravedad
Tabla 2. Criterios de gravedad en pancreatitis post-CPRE

\begin{tabular}{|l|l|l|}
\hline Gravedad & Criterios de Cotton & Criterios de Atlanta \\
\hline Leve & $\begin{array}{l}\text { Hospitalización durante 2-3 } \\
\text { días }\end{array}$ & $\begin{array}{l}\text { Sin F0* ni } \\
\text { complicaciones }^{\dagger} \text { locales }^{\dagger} \text { o sistémicas }\end{array}$ \\
\hline Moderada & Hospitalización de 4-10 días & $\begin{array}{l}\text { F0 transitoria }(<48 \mathrm{~h}) \text { o } \\
\text { complicaciones } \\
\text { locales/sistémicas sin }\end{array}$ \\
\hline Grave & $\begin{array}{l}\text { Hospersistente } \\
\text { pancreatitis hemorrágica, } \\
\text { flemón, pseudoquiste, } \\
\text { necesidad de drenaje } \\
\text { percutáneo o quirúrgico }\end{array}$ & \\
\hline
\end{tabular}

${ }^{*}$ FO de acuerdo con la puntuación de Marshall ( $\left.\geq 2\right)$.

${ }^{\dagger}$ Complicaciones locales: colección líquida peripancreática aguda, pseudoquiste pancreático, colección necrótica aguda y necrosis encapsulada.

${ }^{\ddagger}$ Complicaciones sistémicas: exacerbación de comorbilidades previas (síndrome coronario agudo, enfermedad pulmonar crónica, etc.).

CPRE: Colangiopancreatografía retrógrada endoscópica; FO: falla orgánica. Adaptada de Cotton, et al., $1991^{17}$ y Banks, et al., 2012

está determinada por la duración de la estancia hospitalaria y el desarrollo de complicaciones ${ }^{17}$.

- Criterios de la clasificación de Atlanta (2012). Se requieren dos de los siguientes tres criterios: dolor abdominal pancreático, enzimas pancreáticas $\geq 3$ veces el límite superior normal y/o hallazgos característicos de pancreatitis aguda en un estudio de imagen. En esta clasificación la gravedad se establece de acuerdo con la presencia de falla orgánica y/o complicaciones (Tabla 2$)^{18}$.

La definición del consenso de Cotton es la que se ha utilizado con mayor frecuencia en los estudios clínicos para establecer el diagnóstico, ya que toma en cuenta la presencia de dolor preexistente en pacientes con pancreatitis crónica, así como el momento en el que las enzimas pancreáticas se elevan. Sin embargo, la que establece con mayor precisión la gravedad del cuadro es la clasificación de Atlanta. Smeets, et al. demostraron que los criterios de Atlanta logran reclasificar la gravedad en el $26 \%$ de los casos y también son mejores para predecir mortalidad con respecto a la definición de Cotton (sensibilidad del 100 vs. $55 \%$, especificidad del 98 vs. $72 \%$, valor predictivo positivo del 58 vs. $5 \%$ y valor predictivo negativo del 100 vs. $98 \% ; p<0.001)^{19}$.

\section{Fisiopatogenia}

No se conoce con exactitud la fisiopatología de la PPC. Se ha planteado un modelo multifactorial, donde 
las dos teorías más aceptadas son la del trauma directo y la del daño hidrostático.

- Trauma directo. La manipulación repetida y prolongada de la papila duodenal y el daño térmico que se produce durante una esfinterotomía o una ampulectomía provocan edema de la papila y/o espasmo del esfínter de Oddi. La obstrucción transitoria al flujo de salida del líquido pancreático favorece la activación intracelular de las enzimas proteolíticas. Por otra parte, la canulación recurrente y profunda del conducto pancreático principal incrementa el riesgo de perforación ductal y daño pancreático ${ }^{20}$.

- Daño hidrostático. La cantidad de medio de contraste $(>2 \mathrm{ml})$ y la presión con la que este se inyecta pueden ocasionar ruptura de las membranas celulares y uniones estrechas de las células acinares y ductales. Esto favorece el reflujo del líquido pancreático hacia el espacio intersticial con el daño enzimático subsecuente ${ }^{21-24}$. En fluoroscopia esto se traduce como el signo de acinarización que corresponde a la visualización del medio de contraste en el parénquima pancreático ${ }^{23,25,26}$.

- Daño químico. En un modelo experimental se observó que el medio de contraste puede activar vías de señalización intracelular involucradas en la patogénesis de la pancreatitis $\left(\mathrm{Ca}^{2+}\right.$, calcineurina y factor nuclear kappa B [NF-kB] $)^{24}$. Aunque no se ha demostrado que la osmolaridad del medio de contraste juegue un papel importante ${ }^{27}$, el aclaramiento de este con solución salina durante la CPRE parece reducir la tasa de eventos adversos ${ }^{28}$.

- Factores microbiológicos. Toxinas y enzimas bacterianas pueden quizá desencadenar una respuesta inflamatoria mediada por citocinas o por un efecto citotóxico ${ }^{25,29}$.

\section{Prevención}

\section{Adecuada selección del paciente}

La mejor manera de prevenir complicaciones asociadas a la CPRE es evitando procedimientos innecesarios. Es por ello que siempre se debe corroborar la indicación del estudio (Tabla 1)1,2,30,31. Con el advenimiento de modalidades de imagen menos invasivas, la CPRE ha dejado de ser una herramienta diagnóstica de primera elección y se ha convertido en un procedimiento esencialmente terapéutico ${ }^{31}$. La resonancia magnética y el ultrasonido endoscópico pueden evitar la CPRE en el $30-50 \%$ y en el $36-57 \%$ de los casos, respectivamente $^{32-36}$.
Tabla 3. Factores de riesgo para pancreatitis post-CPRE

\begin{tabular}{|c|c|}
\hline $\begin{array}{l}\text { Relacionados con el } \\
\text { paciente }\end{array}$ & $\begin{array}{l}\text { Relacionados con el } \\
\text { procedimiento }\end{array}$ \\
\hline $\begin{array}{l}\text { Definitivos } \\
\text { - Sexo femenino } \\
\text { - Historia de pancreatitis } \\
\text { aguda } \\
\text { - Antecedente de PPC } \\
\text { - Sospecha de disfunción } \\
\quad \text { del esfínter de Oddi }\end{array}$ & $\begin{array}{l}\text { Definitivos } \\
\text { - Canulación difícil } \\
\text { - Canulación repetida del } \\
\text { conducto pancreático } \\
\text { - Inyección de medio de } \\
\text { contraste en el conducto } \\
\text { pancreático } \\
\text { - Ampulectomía }\end{array}$ \\
\hline $\begin{array}{l}\text { Probables } \\
\text { - Edad* } \\
\text { - Bilirrubinas normales } \\
\text { - Conducto biliar no } \\
\text { dilatado } \\
\text { - Ausencia de } \\
\text { pancreatitis crónica } \\
\text { - Enfermedad renal } \\
\text { crónica }\end{array}$ & $\begin{array}{l}\text { Probables } \\
\text { - Precorte } \\
\text { - Esfinterotomía pancreática } \\
\text { - Dilatación de la papila sin } \\
\text { esfinterotomía previa } \\
\text { - Falla para lograr la } \\
\quad \text { extracción de litos biliares } \\
\text { - US intraductal }\end{array}$ \\
\hline
\end{tabular}

"Edad < 35-40 años.

'Dilatación de corta duración ( $\leq 1$ minuto).

CPRE: colangiopancreatografía retrógrada endoscópica; PPC: pancreatitis post-CPRE; US: ultrasonido.

Adaptada de Dumonceau, et al., $2020^{37}$.

\section{Estratificación del riesgo preprocedimiento}

Actualmente es posible determinar el perfil de riesgo de acuerdo con la presencia o no de distintos factores relacionados con el paciente y el procedimiento (Tabla 3). Mientras algunos factores de riesgo están bien establecidos (definitivos), otros han sido catalogados como «probables» debido a que los resultados obtenidos en los estudios han sido contradictorios. Recientemente la ESGE (European Society of Gastrointestinal Endoscopy) definió como grupo de alto riesgo a aquellos pacientes con al menos un factor definitivo o dos probables ${ }^{37}$.

Algunos estudios han sugerido que la experiencia del endoscopista y el volumen de procedimientos realizados en cada centro se asocian a una menor tasa de procedimientos fallidos y de complicaciones globales, pero no de PPC. Esto probablemente se debe a que los centros con alto volumen atienden una mayor proporción de pacientes con factores de alto riesgo y al mismo tiempo realizan procedimientos más complejos $^{14,16,38,39}$.

Por otro lado, el antecedente de esfinterotomía, el uso de inhibidores de calcineurina, el cáncer de páncreas y la pancreatitis crónica avanzada parecen tener un efecto protector ${ }^{40,41}$. 


\section{Técnica del procedimiento}

\section{Canulación convencional}

De las técnicas de canulación convencional, la asistida con guía metálica es superior a la asistida con medio de contraste en cuanto a tasa de canulación exitosa, menor necesidad de precorte y menor riesgo de pancreatitis (relative risk [RR]: 0.51; intervalo de confianza del 95\% [IC 95\%]: 0.32-0.82 $)^{42}$. Este riesgo también disminuye cuando el manejo de la guía hidrofílica lo realiza el endoscopista mediante un sistema de intercambio rápido en lugar del asistente (2.8 vs. $9.3 \% ; p=0.049)^{43}$.

\section{TÉCNICAS AVANZADAS EN CANULACIÓN DIFÍCIL}

Se considera canulación difícil cuando no se logra acceder al conducto biliar en los primeros 5 minutos después de visualizar la papila, $>5$ intentos de canulación $0>1$ canulación no intencionada del conducto pancreático u opacificación de este con medio de contraste $^{44}$. Estos factores no solamente incrementan el riesgo de PPC (12.3\% con 3 criterios presentes vs. $6.9 \%$ con 1 criterio vs. $2.2 \%$ si están ausentes), sino que además predicen la probabilidad de falla de canulación primaria con técnica convencional (éxito de canulación del $20.4 \%$ con 3 criterios presentes vs. $79.6 \%$ cuando están ausentes; $p<0.001)^{45}$. Dichos hallazgos hacen evidente la utilidad de cambiar a una técnica de canulación avanzada en estos casos. En dos metaanálisis, la canulación con doble guía aumentó el riesgo de PPC en comparación con otras técnicas avanzadas, sin diferencias en la tasa de canulación exitosa ${ }^{46,47}$. Este riesgo se minimiza con la colocación de prótesis pancreáticas (RR: 0.13; IC 95\%: 0.016-0.95; odds ratio

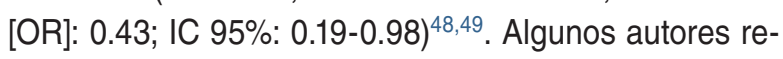
comiendan colocarlas en todo paciente con canulación inadvertida del conducto pancreático ${ }^{37}$, mientras que otros consideran la administración de anti-inflamatorios no esteroideos (AINE) como una alternativa cuando la canulación con doble guía se lleva acabo de forma temprana en pacientes con bajo riesgo $0^{50}$.

El precorte temprano también reduce significativamente los eventos de pancreatitis cuando es realizado por endoscopistas expertos (RR: 0.29-0.57) y parece ser más costo-efectivo que las prótesis pancreáticas (reduciendo los costos en un 29\%) ${ }^{51-53}$. Jang, et al. encontraron que en grupos de alto riesgo el precorte como método de canulación primaria incrementa la tasa de canulación biliar exitosa con menos eventos adversos en comparación con la técnica convencional (canulación: 97.9 vs. $89.7 \%, p=0.005$; pancreatitis: 0 vs. $9.2 \%, p=0.001)^{54}$.

\section{Pancreatografía}

Cuando exista la indicación, se recomienda realizarla con el menor número de inyecciones y la mínima cantidad de contraste posible, evitando la opacificación completa del conducto a menos que sea estrictamente necesario, esto con el objetivo de limitar al máximo el daño pancreático ${ }^{23,55}$. En algunos estudios la opacificación completa del conducto pancreático incrementó el riesgo de PCC en comparación con el grupo de pacientes en quienes únicamente se visualizó el segmento proximal (cabeza) ${ }^{6,56}$. Al final es importante aspirar el medio de contraste residual y valorar el aclaramiento con solución salina ${ }^{23,28}$.

\section{Prótesis plásticas pancreáticas}

Teóricamente favorecen el drenaje pancreático en pacientes con obstrucción transitoria causada por edema de la papila. La colocación de prótesis pancreática se asocia con un menor riesgo de PPC (OR: 0.32; IC 95\%: 0.23-0.45; $p<0.001$ ) y pancreatitis grave (OR: 0.24; IC 95\%: 0.06-0.94; $p=0.04$ ) con un número necesario de pacientes que tratar (NNT) de 7-857-59. Aunque esta intervención es útil en población con riesgo promedio y alto, la estrategia más costo-efectiva es reservar su uso para este último grupo de pacientes ${ }^{58-61}$.

Se sugiere utilizar prótesis modificadas, con un flat externo que evite la migración intraductal y sin flat interno para favorecer la migración espontánea en los siguientes días ${ }^{62}$. Las de $5 \mathrm{Fr}$ son superiores a las de $3 \mathrm{Fr}$ en la profilaxis de pancreatitis ${ }^{63}$, pero no hay una longitud óptima recomendada, ya que los pocos estudios que comparan diferentes longitudes ( 3 vs. $5 \mathrm{~cm}$ ) muestran resultados contradictorios ${ }^{64,65}$. Probablemente, más que una longitud determinada, lo más importante es que el extremo interno de la prótesis quede distal al cuello pancreático (cuerpo o cola). De acuerdo con un estudio previo, si el extremo intraductal está a nivel de la cabeza se incrementa el riesgo de pancreatitis (OR: 3.8; IC 95\%: 1.1-12.9; $p=0.03$ ) Un escenario especial es el grupo de pacientes en quienes se realiza ampulectomía endoscópica, donde las de $7 \mathrm{~cm}$ parecen tener una mayor eficacia comparadas con las de $5 \mathrm{~cm}$ (OR: 0.16; IC 95\%: 0.27-0.94; $p=0.042)^{67}$. 
Los principales inconvenientes de esta estrategia son la necesidad de radiografía abdominal para corroborar la expulsión espontánea en los siguientes 5-10 días ${ }^{37}$, la necesidad de retirar la prótesis vía endoscópica en caso de retención $(4-10 \%)^{68,69}$ y el riesgo incrementado de pancreatitis en colocación fallida (porcentaje de falla: 2.4-19.5\%; riesgo de PPC: $35 \%$; OR: 3.2; IC 95\%: 1.2-8.3) ${ }^{70}$. Por los costos que pueden derivar de esta intervención se ha investigado si los AINE rectales son equivalentes al uso de prótesis en laprofilaxis dePPC. Nohay ensayosaleatorizadosquecomparen el uso de estas dos terapéuticas de forma aislada o combinada, sin embargo en un metaanálisis en red la indometacina fue superior (OR: 0.48; IC 95\%: 0.26$0.87)^{60}$. Esto se contrapone con el resultado de otro metaanálisis donde las prótesis tuvieron mayor efica$\mathrm{cia}^{71}$. De acuerdo con otros estudios la combinación de indometacina más prótesis puede incrementar los costos de forma importante sin aportar un beneficio adicional $^{72,73}$.

\section{Profilaxis farmacológica}

\section{Antinflamatorios no esteroideos}

El mecanismo mediante el cual previenen la PPC no se conoce del todo. Los AINE son inhibidores potentes de la fosfolipasa A2, que parece ser uno de los principales detonantes de la cascada inflamatoria en pancreatitis y constituye además un predictor de falla orgánica múltiple ${ }^{74-76}$. En estudios experimentales la inhibición de esta enzima atenúa la respuesta inflamatoria mediada por citocinas y la gravedad del daño orgánico ${ }^{77,78}$.

Varios metaanálisis han establecido que los AINE reducen la incidencia global de pancreatitis (OR: 0.20.6 ; NNT: 8-21), la incidencia de pancreatitis moderada-grave (RR: 0.3-0.56; NNT: 33-39) y la mortalidad (OR: 0.10$)^{58,79-85}$. Este efecto positivo se ha observado en grupos con riesgo promedio y alto ${ }^{80,83}$. Es importante destacar que son seguros y no se han asociado a un mayor riesgo de eventos adversos cuando se comparan con placebo (OR: 0.8; IC 95\%: 0.47-1.36) ${ }^{86}$. Su amplia disponibilidad, bajo costo, adecuado perfil de seguridad y efecto benéfico en la prevención de PPC han hecho que sea el tratamiento profiláctico de primera línea.

Los únicos AINE que han mostrado beneficio son la indometacina y el diclofenaco por vía rectal ${ }^{85}$. La dosis que se ha empleado en la mayor parte de los estudios y que ha demostrado ser efectiva es la de $100 \mathrm{mg}$, por lo que actualmente se considera la dosis estándar. Sin embargo, en los últimos años se ha cuestionado si esta dosis es la óptima, si dosis altas $(200 \mathrm{mg})$ tienen un efecto aditivo y si dosis bajas $(25-50 \mathrm{mg})$ pudieran tener el mismo beneficio con menos eventos adversos. En un estudio retrospectivo Leerhøy, et al. encontraron que el efecto protector de los AINE pudiera ser menor en pacientes con peso corporal elevado ${ }^{87}$. Hay dos ensayos aleatorizados y doble ciego que han comparado la eficacia de dosis altas de indometacina con respecto a la dosis estándar en pacientes de alto riesgo. En un estudio (Lai, et al., $n=162$ ) se administraron $200 \mathrm{mg}$ divididos en dos dosis (100 mg pre-CPRE y $100 \mathrm{mg}$ post-CPRE), mientras que en el otro (Fogel, et al., $n=1,037$ ) se empleó una dosis escalonada de $150 \mathrm{mg}$ al término de la CPRE y $50 \mathrm{mg}$ adicionales a las 4 horas. En ninguno de ellos se observaron diferencias ni en la incidencia ni en la gravedad de la PPC con dosis altas vs. estándar ${ }^{88,89}$. Algunos estudios (en su mayoría asiáticos) que han utilizado dosis bajas de AINE (diclofenaco 25-50 mg e indometacina $50 \mathrm{mg}$ ) también han demostrado un efecto positivo en la prevención de pancreatitis ${ }^{90-93}$. En un estudio retrospectivo que incluyó 155 pacientes, se encontró que la PPC fue menor en el grupo que recibió $50 \mathrm{mg}$ de diclofenaco comparado con el grupo de $25 \mathrm{mg}$ ( 15.5 vs. $33.3 \%$, $p=0.018$; OR: 0.27, IC 95\%: 0.11-0.70, $p=0.007)^{93}$. Debido a que el beneficio parece ser dependiente de la dosis y que no hay diferencias en la tasa de eventos adversos con dosis bajas vs. estándar, la dosificación más recomendable continúa siendo de $100 \mathrm{mg}$.

Se sugiere suministrarlos 30 minutos antes de la CPRE, no obstante todavía no ha sido posible establecer cuál es el mejor momento para lograr el mayor impacto en la reducción del riesgo de pancreatitis. En algunos metaanálisis la administración de AINE tanto preprocedimiento como posprocedimiento ha resultado útil, mientras que en otros la primera estrategia es la única maniobra efectiva ${ }^{79,80,82,83}$. Solo existen dos estudios prospectivos, comparativos con resultados contradictorios. El estudio más grande (ensayo aleatorizado, multicéntrico, un solo ciego) que incluyó 2,600 pacientes, realizó un subanálisis para evaluar las diferencias entre la administración de indometacina preprocedimiento y posprocedimiento en pacientes con alto riesgo $(n=586)$. La incidencia de PPC fue menor en el grupo que recibió indometacina antes del estudio (6 vs. $3 \%$; $p=0.005)^{94}$. En un segundo estudio comparativo en el que se incluyeron 178 pacientes (con riesgo promedio y alto), el tiempo en el que se administró la indometacina no se asoció con el riesgo de pancreatitis (8.2\% 
preprocedimiento vs. $6.4 \%$ posprocedimiento; OR: 0.65 ; IC 95\%: 0.42-4.04; $p=0.6$ ). En el subanálisis de pacientes con alto riesgo se observó una mayor tasa de pancreatitis en el grupo de indometacina preprocedimiento ( $21.7 \%$ [ $5 / 23$ vs. $12.8 \%$ [5/39), sin embargo estas diferencias no fueron significativas $(p=0.28)^{95}$.

\section{HidRatación agRESIVA CON SOlUCIÓN RINGER LACTATO VS. SOLUCIÓN SALINA $0.9 \%$}

La solución ringer lactato tiene tres beneficios protectores:

1. Previene el daño al mejorar la perfusión microvascular del parénquima pancreático ${ }^{96,97}$.

2. Atenúa la acidosis metabólica y de esta manera limita la activación de las proenzimas ${ }^{98,99}$.

3. Ejerce un efecto antiinflamatorio potente. Modelos experimentales han demostrado que el lactato actúa como un inmunomodulador de la respuesta inflamatoria al inhibir la activación de la vía de señalización NF-kB, reduciendo la expresión del factor de necrosis tumoral $\alpha$ y la interleucina $1 \beta$ y previniendo la inhibición del receptor de manosa $\mathrm{C}$ tipo 1 localizado en la superficie de macrófagos con fenotipo inflamatorio ${ }^{100,101}$. En un ensayo clínico aleatorizado también se observó una reducción significativa de la respuesta inflamatoria sistémica y de los niveles de proteína $C$ reactiva a las 24 horas de iniciada la reanimación con solución Ringer lactato $(\mathrm{RL})$ con respecto al grupo de solución salina (SS) $0.9 \%{ }^{99}$.

La hidratación agresiva (volumen $\geq 3 \mathrm{I}$ ) reduce la incidencia de PPC (OR: 0.44; IC 95\%: 0.28-0.69; $p=$ 0.0004 ; NNT: 17$)^{102}$, disminuye la incidencia de pancreatitis moderada-grave (OR: 0.16; IC 95\%: 0.03$0.96)^{103}$ y acorta los días de estancia hospitalaria, sin diferencia en la tasa de eventos adversos cuando se compara con hidratación estándar ${ }^{102-104}$. Este efecto protector es dependiente del volumen, del tipo de solución empleada y probablemente del tiempo en el cual se administra. En un estudio de cohorte retrospectivo, DiMagno, et al. incluyeron 6,505 pacientes en quienes se realizaron 8,264 CPRE, encontrando que el volumen total infundido periprocedimiento fue significativamente mayor en el grupo de pancreatitis leve con respecto al grupo de pancreatitis moderada-grave $(3.3 \pm 1.9$ vs. $5.5 \pm 2.5$ l; $p<0.0001$ ). En el análisis multivariante la hidratación intensa fue un factor protector independiente de gravedad (OR: 0.20; IC 95\%: 0.05-0.83) ${ }^{105}$. En otro estudio (aleatorizado, multicéntrico, $\mathrm{n}=395$ ) en el que se compararon dos tipos de soluciones, se observó que la tasa de PPC fue menor en el grupo de hidratación agresiva con $\mathrm{RL}$ en comparación con la hidratación estándar (3 vs. 11.6\%; RR: 0.26; IC 95\%: $0.08-0.76 ; p=0.008$ ). Por otra parte, la hidratación agresiva con SS $0.9 \%$ no fue superior a la hidratación estándar en la prevención de pancreatitis (6.7 vs. 11.6\%; RR: 0.57; IC 95\%: 0.26-1.27; $p=0.17)^{106}$.

Se han propuesto diferentes esquemas de hidratación, pero no ha sido posible dilucidar cuál es el volumen más efectivo. El protocolo más empleado y recomendado por las guías de la ESGE consiste en una infusión de $3 \mathrm{ml} / \mathrm{kg} / \mathrm{h}$ durante el procedimiento, seguido de un bolo de $20 \mathrm{ml} / \mathrm{kg}$ al término del estudio y una infusión de $3 \mathrm{ml} / \mathrm{kg} / \mathrm{h}$ durante las siguientes $8 \mathrm{~h}^{37,107}$. El tiempo de infusión prolongado hace que esta terapia esté restringida al grupo de sujetos hospitalizados. Por otra parte, esta maniobra terapéutica está contraindicada en pacientes con riesgo de sobrecarga (insuficiencia cardiaca, enfermedad renal, cirrosis, insuficiencia respiratoria). En México no hay RL, por lo que una alternativa es el uso de solución Hartmann.

\section{HIDRATACIÓN AGRESIVA + ANTIINFLAMATORIOS NO ESTEROIDEOS RECTALES}

Es factible que esta combinación pueda tener un efecto sinérgico, ya que tienen mecanismos protectores distintos. Hasta el momento hay tres estudios que han evaluado el efecto aditivo de los AINE (diclofenaco e indometacina) y la hidratación agresiva (RL y SS 0.9\%), sin embargo los resultados son discordantes. Un ensayo aleatorizado, controlado con placebo y doble ciego que incluyó 192 pacientes con alto riesgo de PPC encontró que la combinación de $\mathrm{RL}$ + indometacina rectal fue superior al uso de SS $0.9 \%$ en la prevención de esta complicación (3 vs. $21 \%$; $p=0.04$; reducción del riesgo absoluto del $14.6 \%$; reducción del riesgo relativo del $70 \%$ ) y en la reducción de readmisiones hospitalarias ( 2 vs. $13 \% ; p=0.03$ ) con un NNT de $6.9^{108}$. Es importante destacar que el riesgo de pancreatitis en el grupo de $\mathrm{RL}+$ placebo fue relativamente alto $(19 \%)$ y muy similar al del grupo de SS $0.9 \%$ + placebo $(21 \%)$, esto probablemente porque el volumen de hidratación empleado fue significativamente menor al usado en estudios previos ${ }^{108}$. En otro ensayo aleatorizado ( $n=406$ ) se observó que la hidratación agresiva con SS $0.9 \%$ + indometacina fue superior a la monoterapia con indometacina para reducir los eventos de pancreatitis en pacientes con riesgo promedio (reducción del riesgo absoluto del 5.2 vs. $16.2 \%$ y del riesgo relativo del 32 vs. $100 \%)^{109}$. En un tercer estudio $(n=219)$ la combinación de diclofenaco rectal + hidratación intensa con $R L$ no demostró tener ningún beneficio adicional a la monoterapia con AINE ${ }^{110}$. 


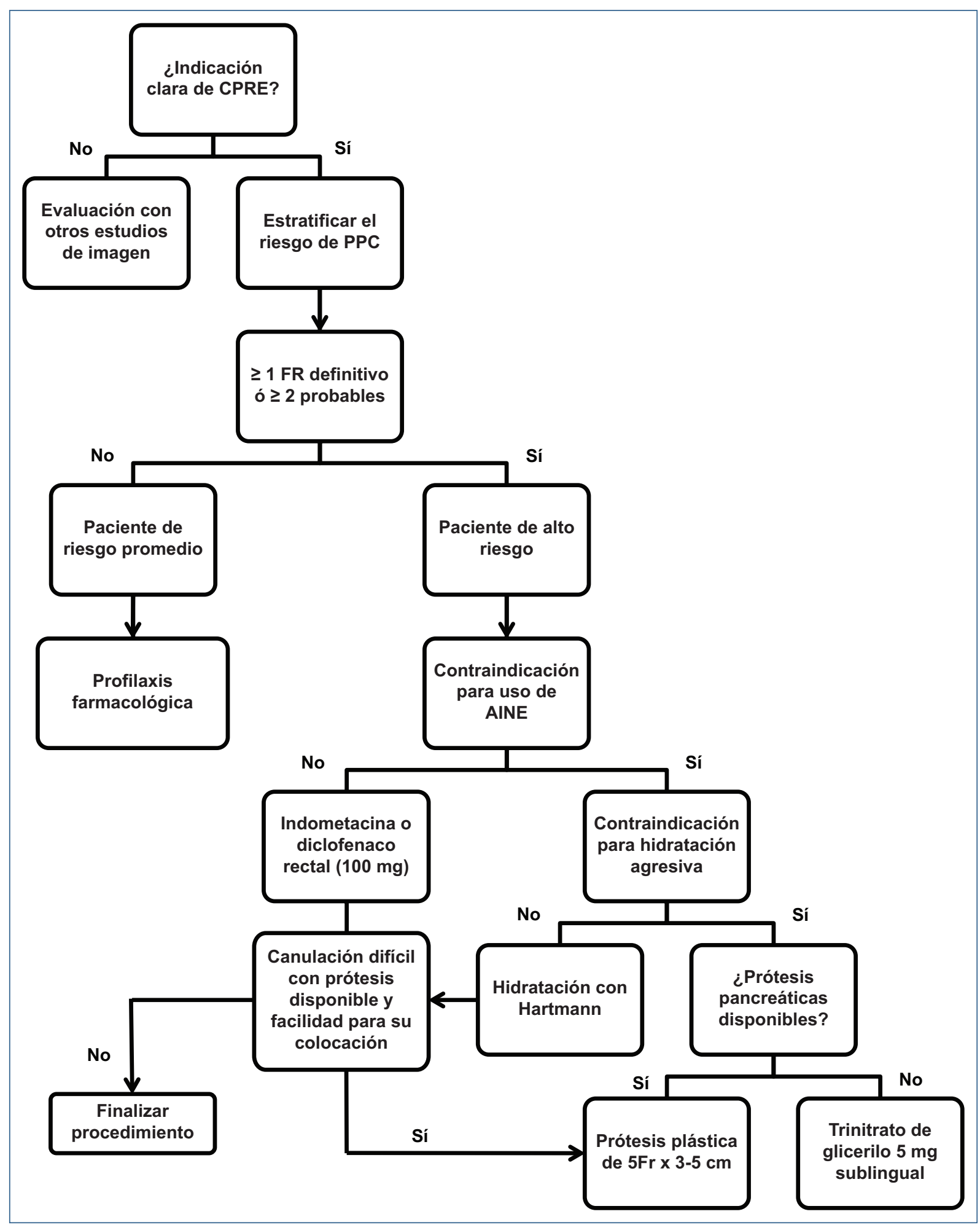

Figura 1. Algoritmo para la prevención de pancreatitis post-CPRE.

CPRE: colangiopancreatografía retrógrada endoscópica; PPC: pancreatitis post-CPRE; AINE: antiinflamatorios no esterideos; FR: factores de riesgo.

Facilidad para la colocación de una prótesis pancreática: cuando se tenga una guía en el conducto pancreático. Facilidad para la colocación de una prótesis pancreática: cuando se tenga una guía en el conducto pancreático. 


\section{Nitratos sublinguales}

Se ha postulado que inducen la relajación del esfínter de Oddi111, favoreciendo el drenaje pancreático. En un metaanálisis (12 ensayos aleatorizados, $n=2,649$ ) los nitratos lograron reducir la incidencia de pancreatitis (RR: 0.67; IC 95\%: 0.52-0.87), pero no la gravedad $^{112}$. La combinación de nitratos + AINE (indometacina o diclofenaco) ha demostrado ser superior a los AINE solos para reducir el riesgo de PPC y de pancreatitis moderada-grave, con un NNT de 12-26113,114. Sin embargo, estos fármacos pueden causar eventos adversos importantes como cefalea (4\%) e hipotensión $(54 \%)^{112}$. Por ello se ha sugerido la administración de $5 \mathrm{mg}$ de trinitrato de glicerilo sublingual preprocedimiento únicamente en pacientes con riesgo alto cuando estén contraindicados los AINE y la hidratación agresiva $^{37}$.

\section{EPINEFRINA}

Luo, et al., en un ensayo clínico aleatorizado, multicéntrico y doble ciego compararon la eficacia del uso de indometacina rectal + epinefrina tópica sobre la papila $(n=576)$ vs. indometacina rectal sola $(n=582)$. El estudio se finalizó de forma temprana debido a que en un análisis interino se encontró un mayor riesgo de pancreatitis en el grupo de terapia combinada con respecto al grupo de monoterapia con indometacina rectal (8.5 vs. $5.3 \%$; RR: 1.60; IC 95\%: 1.03-2.47; $p=0.033)^{115}$. En un metaanálisis reciente en el que se incluyeron este y otros dos ensayos aleatorizados con un total de 2,224 pacientes no se encontraron diferencias en la incidencia de pancreatitis (7.3 vs. 4.8\%; RR: 1.15; IC 95\%: 0.62-2.22) ni en la tasa de eventos adversos (9.9 vs. 7.8\%; RR: 1.3; IC 95\%: 0.93$1.7 ; p=0.13)$ y tampoco hubo diferencias en mortalidad (0.3 vs. $0.4 \%$; RR: 0.85 ; IC $95 \%$ : 0.22-3.24; $p=0.81$ ) entre ambos grupos. Con la evidencia actual ya no se recomienda utilizarla ${ }^{116}$.

\section{Conclusión}

En los últimos años se ha investigado la utilidad de diferentes intervenciones en la prevención de PPC de acuerdo con el riesgo de cada paciente, no obstante solo tres de ellas han demostrado un beneficio irrefutable, que son: colocación de prótesis plásticas pancreáticas, AINE e hidratación agresiva. En la figura 1 se presenta un algoritmo para facilitar la toma de decisiones.

\section{Financiamiento}

La presente investigación no ha recibido ayudas específicas provenientes de agencias del sector público, sector comercial o entidades sin ánimo de lucro.

\section{Conflicto de intereses}

Los autores declaran no tener conflicto de intereses.

\section{Responsabilidades éticas}

Protección de personas y animales. Los autores declaran que para esta investigación no se han realizado experimentos en seres humanos ni en animales.

Confidencialidad de los datos. Los autores declaran que en este artículo no aparecen datos de pacientes.

Derecho a la privacidad y consentimiento informado. Los autores declaran que en este artículo no aparecen datos de pacientes.

\section{Bibliografía}

1. Solomon S, Baillie J. Indications for and contraindications to ERCP. En: Baron TH, Kozarek RA, Carr-Locke DL, editores. ERCP. 3th ed. Philadelphia: Elsevier; 2019. pp. 54-8.

2. Easler JJ, Fogel EL, Sherman S. Cholangiography and pancreatography. En: Chandrasekhara V, Elmunzer BJ, Khashab MA, Muthusamy VR, editores. Clinical Gastrointestinal Endoscopy. 3th ed. Philadelphia: Elsevier; 2019. pp. 538-62

3. Ahmed M, Kanotra R, Savani GT, Kotadiya F, Patel N, Tareen S, et al. Utilization trends in inpatient endoscopic retrograde cholangiopancreatography (ERCP): A cross-sectional US experience. Endosc Int Open. 2017:5(4):E261-E71.

4. Rustagi T, Jamidar PA. Endoscopic retrograde cholangiopancreatography-related adverse events: general overview. Gastrointest Endosc Clin N Am. 2015;25(1):97-106.

5. Masci E, Toti G, Mariani A, Curioni S, Lomazzi A, Dinelli M, et al. Complications of diagnostic and therapeutic ERCP: a prospective multicenter study. Am J Gastroenterol. 2001;96(2):417-23.

6. Vandervoort J, Soetikno RM, Tham TC, Wong RC, Ferrari AP Jr, Montes $\mathrm{H}$, et al. Risk factors for complications after performance of ERCP. Gastrointest Endosc. 2002;56(5):652-6.

7. Christensen M, Matzen P, Schulze S, Rosenberg J. Complications of ERCP: a prospective study. Gastrointest Endosc. 2004;60(5):721-31.

8. Williams EJ, Taylor S, Fairclough P, Hamlyn A, Logan RF, Martin D, et al. Risk factors for complication following ERCP; results of a large-scale, prospective multicenter study. Endoscopy. 2007;39(9):793-801.

9. Andriulli A, Loperfido S, Napolitano G, Niro G, Valvano MR, Spirito F, et al. Incidence rates of post-ERCP complications: a systematic survey of prospective studies. Am J Gastroenterol. 2007;102(8):1781-8.

10. Wang P, Li ZS, Liu F, Ren X, Lu NH, Fan ZN, et al. Risk factors for ERCP-related complications: a prospective multicenter study. Am J Gastroenterol. 2009;104(1):31-40.

11. Cotton PB, Garrow DA, Gallagher J, Romagnuolo J. Risk factors for complications after ERCP: a multivariate analysis of 11,497 procedures over 12 years. Gastrointest Endosc. 2009;70(1):80-8.

12. Jeurnink SM, Siersema PD, Steyerberg EW, Dees J, Poley JW, Haringsma J, et al. Predictors of complications after endoscopic retrograde cholangiopancreatography: a prognostic model for early discharge. Surg Endosc. 2011;25(9):2892-900.

13. Siiki A, Tamminen A, Tomminen $T$, Kuusanmaki $P$. ERCP procedures in a Finnish community hospital: a retrospective analysis of 1207 cases. Scand J Surg. 2012;101(1):45-50.

14. Lee HJ, Cho CM, Heo J, Jung MK, Kim TN, Kim KH, et al. Impact of hospital volume and the experience of endoscopist on adverse events related to endoscopic retrograde cholangiopancreatography: A prospective observational study. Gut Liver. 2020;14(2):257-64.

15. Kochar B, Akshintala VS, Afghani E, Elmunzer BJ, Kim KJ, Lennon AM, et al. Incidence, severity, and mortality of post-ERCP pancreatitis: a 
systematic review by using randomized, controlled trials. Gastrointest Endosc. 2015;81(1):143-9. e9.

16. Testoni PA, Mariani A, Giussani A, Vailati C, Masci E, Macarri G, et al. Risk factors for post-ERCP pancreatitis in high- and low-volume centers and among expert and non-expert operators: a prospective multicenter study. Am J Gastroenterol. 2010;105(8):1753-61.

17. Cotton PB, Lehman G, Vennes J, Geenen JE, Russell RC, Meyers WC, et al. Endoscopic sphincterotomy complications and their management: an attempt at consensus. Gastrointest Endosc. 1991;37(3):383-93.

18. Banks PA, Bollen TL, Dervenis C, Gooszen HG, Johnson CD, Sarr MG, et al. Classification of acute pancreatitis--2012: revision of the Atlanta classification and definitions by international consensus. Gut. 2013;62(1):102-11.

19. Smeets X, Bouhouch N, Buxbaum J, Zhang H, Cho J, Verdonk RC, et al. The revised Atlanta criteria more accurately reflect severity of post-ERCP pancreatitis compared to the consensus criteria. United European Gastroenterol J. 2019;7(4):557-64.

20. Zouhairi ME, Swartz D, Shah T. Post-ERCP pancreatitis: Mechanisms, risk factors, and prevention. Pancreatic Dis Ther. 2013;3(2):116.

21. Bockman DE, Schiller WR, Anderson MC. Route of retrograde flow in the exocrine pancreas during ductal hypertension. Arch Surg. 1971;103(2):321-9.

22. Waldron RL 2nd, Luse SA, Wollowick HE, Seaman WB. Demonstration of a retrograde pancreatic pathway: correlation of roentgenographic and electron microscopic studies. Am J Roentgenol Radium Ther Nucl Med. 1971:111(4):695-9.

23. Abdel Aziz AM, Lehman GA. Pancreatitis after endoscopic retrograde cholangio-pancreatography. World J Gastroenterol. 2007;13(19): 2655-68.

24. Jin S, Orabi Al, Le T, Javed TA, Sah S, Eisses JF, et al. Exposure to radiocontrast agents induces pancreatic inflammation by activation of nuclear factor-kB, calcium signaling, and calcineurin. Gastroenterology. 2015;149(3):753-64. e11.

25. Pezzilli R, Romboli E, Campana D, Corinaldesi R. Mechanisms involved in the onset of post-ERCP pancreatitis. JOP. 2002;3(6):162-8.

26. Katsinelos P, Lazaraki G, Chatzimavroudis G, Gkagkalis S, Vasiliadis I, Papaeuthimiou A, et al. Risk factors for therapeutic ERCP-related complications: an analysis of 2,715 cases performed by a single endoscopist. Ann Gastroenterol. 2014;27(1):65-72.

27. George S, Kulkarni AA, Stevens G, Forsmark CE, Draganov P. Role of osmolality of contrast media in the development of post-ERCP pancreatitis: a metanalysis. Dig Dis Sci. 2004;49(3):503-8.

28. Ökmen H, Gürbulak B, Düzköylü Y, Gürbulak EK. The effect of post-contrast washıng on post-endoscopıc retrograde cholangıopancreatography pancreatitis. Int J Clin Exp Med. 2016;9(8):15868-75.

29. Keynes WM. A nonpancreatic source of the proteolytic-enzyme amidase and bacteriology in experimental acute pancreatitis. Ann Surg. 1980;191(2):187-99.

30. Freeman ML. Complications of endoscopic retrograde cholangiopancreatography: avoidance and management. Gastrointest Endosc Clin N Am. 2012;22(3):567-86

31. Boškoski I, Costamagna G. How to prevent post-endoscopic retrograde cholangiopancreatography pancreatitis. Gastroenterology. 2020;158(8):P2037-40.

32. Kaltenthaler EC, Walters SJ, Chilcott J, Blakeborough A, Vergel YB, Thomas $\mathrm{S}$. MRCP compared to diagnostic ERCP for diagnosis when biliary obstruction is suspected: a systematic review. BMC Med Imaging. 2006;6:9.

33. Bhat M, Romagnuolo J, da Silveira E, Reinhold C, Valois E, Martel M, et al. Randomised clinical trial: MRCP-first vs. ERCP-first approach in patients with suspected biliary obstruction due to bile duct stones. Aliment Pharmacol Ther. 2013;38(9):1045-53.

34. Zaheer A, Anwar MM, Donohoe C, O'Keeffe S, Mushtaq H, Kelleher B, et al. The diagnostic accuracy of endoscopic ultrasound in suspected biliary obstruction and its impact on endoscopic retrograde cholangiopancreatography burden in real clinical practice: a consecutive analysis. Eur J Gastroenterol Hepatol. 2013;25(7):850-7.

35. Sharma R, Menachery J, Choudhary NS, Kumar M, Puri R, Sud R. Routine endoscopic ultrasound in moderate and indeterminate risk patients of suspected choledocholithiasis to avoid unwarranted ERCP: A prospective randomized blinded study. Indian J Gastroenterol. 2015;34(4):300-4.

36. Patel R, Ingle M, Choksi D, Poddar P, Pandey V, Sawant P. Endoscopic ultrasonography can prevent unnecessary diagnostic endoscopic retrograde cholangiopancreatography even in patients with high likelihood of choledocholithiasis and inconclusive ultrasonography: Results of a prospective study. Clin Endosc. 2017;50(6):592-7.

37. Dumonceau JM, Kapral C, Aabakken L, Papanikolaou IS, Tringali A, Vanbiervliet G, et al. ERCP-related adverse events: European Society of Gastrointestinal Endoscopy (ESGE) Guideline. Endoscopy. 2020;52(2):127-49.

38. Coté GA, Imler TD, Xu H, Teal E, French DD, Imperiale TF, et al. Lower provider volume is associated with higher failure rates for endoscopic retrograde cholangiopancreatography. Med Care. 2013:51(12):1040-7.

39. Keswani RN, Qumseya BJ, O'Dwyer LC, Wani S. Association between endoscopist and center endoscopic retrograde cholangiopancreatography volume with procedure success and adverse outcomes: A systematic review and meta-analysis. Clin Gastroenterol Hepatol. 2017;15(12):186675 e3.

40. Elmunzer BJ. Reducing the risk of post-endoscopic retrograde cholangiopancreatography pancreatitis. Dig Endosc. 2017;29(7):749-57.

41. Thiruvengadam NR, Forde KA, Chandrasekhara V, Ahmad NA, Ginsberg GG, Khungar V, et al. Tacrolimus and indomethacin are safe and effective at reducing pancreatitis after endoscopic retrograde cholangiopancreatography in patients who have undergone liver transplantation. Clin Gastroenterol Hepatol. 2020;18(5):1224-32. e1.

42. Tse F, Yuan Y, Moayyedi P, Leontiadis GI. Guidewire-assisted cannulation of the common bile duct for the prevention of post-endoscopic retrograde cholangiopancreatography (ERCP) pancreatitis. Cochrane Database Syst Rev. 2012;12:CD009662.

43. Buxbaum J, Leonor P, Tung J, Lane C, Sahakian A, Laine L. Randomized trial of endoscopist-controlled vs. assistant-controlled wire-guided cannulation of the bile duct. Am J Gastroenterol. 2016;111(12):1841-7.

44. Halttunen J, Meisner S, Aabakken L, Arnelo U, Grönroos J, Hauge T, et al. Difficult cannulation as defined by a prospective study of the Scandinavian Association for Digestive Endoscopy (SADE) in 907 ERCPs. Scand J Gastroenterol. 2014;49(6):752-8.

45. Ismail S, Udd M, Lindstrom O, Rainio M, Halttunen J, Kylanpaa L. Criteria for difficult biliary cannulation: start to count. Eur J Gastroenterol Hepatol. 2019;31(10):1200-5.

46. Tse F, Yuan Y, Moayyedi P, Leontiadis GI, Barkun AN. Double-guidewire technique in difficult biliary cannulation for the prevention of post-ERCP pancreatitis: a systematic review and meta-analysis. Endoscopy. 2017;49(1):15-26.

47. Kanth R, Gonzalez Z, Roy N, Karna N, Chitnavis V, Roy P. Double guide-wire technique for difficult biliary cannulation: Meta-Analysis of randomized controlled trials. Gastrointest Endosc. 2019;89(6):AB573. Abstract.

48. Ito K, Fujita N, Noda Y, Kobayashi G, Obana T, Horaguchi J, et al. Can pancreatic duct stenting prevent post-ERCP pancreatitis in patients who undergo pancreatic duct guidewire placement for achieving selective biliary cannulation? A prospective randomized controlled trial. J Gastroenterol. 2010;45(11):1183-91.

49. Phillip V, Pukitis A, Epstein A, Hapfelmeier A, Haf D, Schwab M, et al. Pancreatic stenting to prevent post-ERCP pancreatitis: a randomized multicenter trial. Endosc Int Open. 2019;7(7):E860-E8.

50. Leerhøy B, Elmunzer BJ. How to avoid post-endoscopic retrograde cholangiopancreatography pancreatitis. Gastrointest Endosc Clin N Am. 2018;28(4):439-54.

51. Sundaralingam P, Masson P, Bourke MJ. Early precut sphincterotomy does not increase risk during endoscopic retrograde cholangiopancreatography in patients with difficult biliary access: A meta-analysis of randomized controlled trials. Clin Gastroenterol Hepatol. 2015; 13(10): $1722-9$ e2.

52. Tang Z, Yang Y, Yang Z, Meng W, Li X. Early precut sphincterotomy does not increase the risk of adverse events for patients with difficult biliary access: A systematic review of randomized clinical trials with meta-analysis and trial sequential analysis. Medicine (Baltimore). 2018; 97(36): e12213.

53. Hwang HJ, Guidi MA, Curvale C, Lasa J, Matano R. Post-ERCP pancreatitis: early precut or pancreatic duct stent? A multicenter, randomized-controlled trial and cost-effectiveness analysis. Rev Esp Enferm Dig. 2017:109(3):174-9.

54. Jang SI, Kim DU, Cho JH, Jeong S, Park JS, Lee DH, et al. Primary needle-knife fistulotomy versus conventional cannulation method in a high-risk cohort of post-endoscopic retrograde cholangiopancreatography pancreatitis. Am J Gastroenterol. 2020:115(4):616-24.

55. Haciahmetoglu T, Ertekin C, Dolay K, Yanar F, Yanar H, Kapran Y. The effects of contrast agent and intraductal pressure changes on the development of pancreatitis in an ERCP model in rats. Langenbecks Arch Surg. 2008;393(3):367-72.

56. Cheon YK, Cho KB, Watkins JL, McHenry L, Fogel EL, Sherman S, et al. Frequency and severity of post-ERCP pancreatitis correlated with extent of pancreatic ductal opacification. Gastrointest Endosc. 2007; 65(3):385-93

57. Sugimoto M, Takagi T, Suzuki R, Konno N, Asama H, Sato $\mathrm{Y}$, et al. Pancreatic stents to prevent post-endoscopic retrograde cholangiopancreatography pancreatitis: A meta-analysis. World J Meta-Anal. 2019;7(5):249-58.

58. Vadalà di Prampero SF, Faleschini G, Panic N, Bulajic M. Endoscopic and pharmacological treatment for prophylaxis against postendoscopic retrograde cholangiopancreatography pancreatitis: a meta-analysis and systematic review. Eur J Gastroenterol Hepatol. 2016;28(12):1415-24.

59. Choudhary A, Bechtold ML, Arif M, Szary NM, Puli SR, Othman MO, et al. Pancreatic stents for prophylaxis against post-ERCP pancreatitis: a meta-analysis and systematic review. Gastrointest Endosc. 2011; 73(2):275-82.

60. Akbar A, Abu Dayyeh BK, Baron TH, Wang Z, Altayar O, Murad MH. Rectal nonsteroidal anti-inflammatory drugs are superior to pancreatic duct stents in preventing pancreatitis after endoscopic retrograde cho- 
langiopancreatography: a network meta-analysis. Clin Gastroentero Hepatol. 2013;11(7):778-83

61. Das A, Singh P, Sivak MV Jr, Chak A. Pancreatic-stent placement for prevention of post-ERCP pancreatitis: a cost-effectiveness analysis. Gastrointest Endosc. 2007;65(7):960-8.

62. He Q, Wang L, Peng C, Zou X, Zhan Q, Xu Y, et al. Modified prophylactic 5 -fr pancreatic duct stent enhances the rate of spontaneous dislodgement: A multicenter randomized controlled trial. United European Gastroenterol J. 2018;6(10):1519-26.

63. Afghani E, Akshintala VS, Khashab MA, Law JK, Hutfless SM, Kim KJ, et al. 5-Fr vs. 3-Fr pancreatic stents for the prevention of post-ERCP pancreatitis in high-risk patients: a systematic review and network meta-analysis. Endoscopy. 2014;46(7):573-80

64. Fujisawa T, Kagawa K, Ochiai K, Hisatomi K, Kubota K, Sato H, et al. Prophylactic efficacy of $3-$ or $5-\mathrm{cm}$ pancreatic stents for preventing post-ERCP pancreatitis: A prospective, randomized trial. J Clin Gastroenterol. 2016;50(3):e30-4.

65. Olsson G, Lübbe J, Arnelo U, Jonas E, Törnqvist B, Lundell L, et al. The impact of prophylactic pancreatic stenting on post-ERCP pancreatitis: A nationwide register-based study. United European Gastroenterol J. 2017;5(1):111-8.

66. Sugimoto $M$, Takagi $T$, Suzuki R, Konno N, Asama $H$, Sato $Y$, et al. Pancreatic stents for the prevention of post-endoscopic retrograde cholangiopancreatography pancreatitis should be inserted up to the pancreatic body or tail. World J Gastroenterol. 2018;24(22):2392-9.

67. Minami K, Iwasaki E, Kawasaki S, Fukuhara S, Seino T, Katayama T, et al. A long $(7 \mathrm{~cm})$ prophylactic pancreatic stent decreases incidence of post-endoscopic papillectomy pancreatitis: a retrospective study. Endosc Int Open. 2019;7(12):E1663-E70.

68. Sofuni A, Maguchi H, Itoi T, Katanuma A, Hisai H, Niido T, et al. Prophylaxis of post-endoscopic retrograde cholangiopancreatography pancreatitis by an endoscopic pancreatic spontaneous dislodgement stent. Clin Gastroenterol Hepatol. 2007:5(11):1339-46.

69. Zolotarevsky E, Fehmi SM, Anderson MA, Schoenfeld PS, Elmunzer BJ, Kwon RS, et al. Prophylactic 5-Fr pancreatic duct stents are superior to 3-Fr stents: a randomized controlled trial. Endoscopy. 2011:43(4):325-30.

70. Choksi NS, Fogel EL, Cote GA, Romagnuolo J, Elta GH, Scheiman JM et al. The risk of post-ERCP pancreatitis and the protective effect of rectal indomethacin in cases of attempted but unsuccessful prophylactic pancreatic stent placement. Gastrointest Endosc. 2015;81(1):150-5.

71. Njei B, McCarty TR, Muniraj T, Sharma P, Jamidar PA, Aslanian HR, et al. Comparative effectiveness of pharmacologic and endoscopic interventions for prevention of post-ERCP pancreatitis: a network meta-analysis. Endosc Int Open. 2020;8(1):E29-E40.

72. Sotoudehmanesh R, Ali-Asgari A, Khatibian M, Mohamadnejad M, Merat S, Sadeghi A, et al. Pharmacological prophylaxis versus pancreatic duct stenting plus pharmacological prophylaxis for prevention of post-ERCP pancreatitis in high risk patients: a randomized trial. Endoscopy. 2019;51(10):915-21.

73. Elmunzer BJ, Higgins PD, Saini SD, Scheiman JM, Parker R A, Chak A et al. Does rectal indomethacin eliminate the need for prophylactic pancreatic stent placement in patients undergoing high-risk ERCP? Post hoc efficacy and cost-benefit analyses using prospective clinical trial data. Am J Gastroenterol. 2013;108(3):410-5.

74. Mäkelä A, Kuusi T, Schröder T. Inhibition of serum phospholipase-A2 in acute pancreatitis by pharmacological agents in vitro. Scand J Clin Lab Invest. 1997:57(5):401-7.

75. Miura M, Endo S, Kaku LL, Inoue Y, Sato N, Wakabayshi G, et al. Plasma type II phospholipase A2 levels in patients with acute pancreatitis. Res Commun Mol Pathol Pharmacol. 2001;109:159-64.

76. Aufenanger J, Samman M, Quintel M, Fassbender K, Zimmer W, Bertsch T. Pancreatic phospholipase A2 activity in acute pancreatitis: A prognostic marker for early identification of patients at risk. Clin Chem Lab Med. 2002;40(3):293-7.

77. Zhang KJ, Zhang DL, Jiao XL, Dong C. Effect of phospholipase A2 silencing on acute experimental pancreatitis. Eur Rev Med Pharmacol Sci. 2013:17(24):3279-84

78. Tomita Y, Kuwabara K, Furue S, Tanaka K, Yamada K, Ueno M, et al Effect of a selective inhibitor of secretory phospholipase A2, S-5920/ LY $315920 \mathrm{Na}$, on experimental acute pancreatitis in rats. J Pharmacol Sci. 2004:96(2):144-54.

79. Yaghoobi M, Alzahrani MA, McNabb-Baltar J, Martel M, Barkun AN Rectal indomethacin prevents moderate to severe post-ERCP pancreatitis and death and should be used before the procedure: A meta-analysis of aggregate subgroup data. J Can Assoc Gastroenterol. 2018 1(2):67-75

80. Yu LM, Zhao KJ, Lu B. Use of NSAIDs via the rectal route for the prevention of pancreatitis after ERCP in all-risk patients: An updated meta-analysis. Gastroenterol Res Pract. 2018;2018:1027530.

81. Sajid MS, Khawaja AH, Sayegh M, Singh KK, Philipose Z. Systematic review and meta-analysis on the prophylactic role of non-steroidal anti-inflammatory drugs to prevent post-endoscopic retrograde cholangiopancreatography pancreatitis. World J Gastrointest Endosc. 2015; $7(19): 1341-9$
82. Wan J, Ren Y, Zhu Z, Xia L, Lu N. How to select patients and timing for rectal indomethacin to prevent post-ERCP pancreatitis: a systematic review and meta-analysis. BMC Gastroenterol. 2017;17(1):43.

83. Puig I, Calvet X, Baylina M, Isava Á, Sort P, Llaó J, et al. How and when should NSAIDs be used for preventing post-ERCP pancreatitis? A systematic review and meta-analysis. PLoS One. 2014;9(3):e92922.

84. Ahmad D, Lopez KT, Esmadi MA, Oroszi G, Matteson-Kome ML, Choudhary $A$,et al. The effect of indomethacin in the prevention of post-endoscopic retrograde cholangiopancreatography pancreatitis: a meta-analysis. Pancreas. 2014;43(3):338-42.

85. Serrano JPR, de Moura DTH, Bernardo WM, Ribeiro IB, Franzini TP, de Moura ETH, et al. Nonsteroidal anti-inflammatory drugs versus placebo for post-endoscopic retrograde cholangiopancreatography pancreatitis: a systematic review and meta-analysis. Endosc Int Open. 2019; 7(4):E477-E86.

86. Hou YC, Hu Q, Huang J, Fang JY, Xiong H. Efficacy and safety of rectal nonsteroidal anti-inflammatory drugs for prophylaxis against post-ERCP pancreatitis: a systematic review and meta-analysis. Sci Rep. 2017; 7:46650.

87. Leerhøy B, Nordholm-Carstensen A, Novovic S, Hansen MB, Jørgensen LN. Effect of body weight on fixed dose of diclofenac for the prevention of post-endoscopic retrograde cholangiopancreatography pancreatitis. Scand J Gastroenterol. 2016;51(8):1007-12.

88. Lai JH, Hung CY, Chu CH, Chen CJ, Lin HH, Lin HJ, et al. A randomized trial comparing the efficacy of single-dose and double-dose administration of rectal indomethacin in preventing post-endoscopic retrograde cholangiopancreatography pancreatitis. Medicine (Baltimore). 2019; 98(20):e15742.

89. Fogel EL, Lehman GA, Tarnasky P, Cote GA, Schmidt SE, Waljee AK, et al. Rectal indometacin dose escalation for prevention of pancreatitis after endoscopic retrograde cholangiopancreatography in high-risk patients: a double-blind, randomised controlled trial. Lancet Gastroenterol Hepatol. 2020;5(2):132-41.

90. Elmunzer BJ, Scheiman JM, Lehman GA, Chak A, Mosler P, Higgins PD, et al. A randomized trial of rectal indomethacin to prevent post-ERCP pancreatitis. N Engl J Med. 2012;366(15):1414-22.

91. Otsuka T, Kawazoe S, Nakashita S, Kamachi S, Oeda S, Sumida C, et al. Low-dose rectal diclofenac for prevention of post-endoscopic retrograde cholangiopancreatography pancreatitis: a randomized controlled trial. J Gastroenterol. 2012;47(8):912-7.

92. Okuno M, Shiroko J, Taguchi D, Yamaguchi K, Takada J, Imai S, et al. The effectiveness of the rectal administration of low-dose diclofenac for the prevention of post-endoscopic retrograde cholangiopancreatography pancreatitis. Intern Med. 2018;57(16):2289-94

93. Yoshihara T, Horimoto M, Kitamura T, Osugi N, Ikezoe T, Kotani K, et al. $25 \mathrm{mg}$ versus $50 \mathrm{mg}$ dose of rectal diclofenac for prevention of post-ERCP pancreatitis in Japanese patients: a retrospective study. BMJ Open. 2015;5(3):e006950.

94. Luo $\mathrm{H}$, Zhao L, Leung J, Zhang R, Liu Z, Wang X et al. Routine pre-procedural rectal indometacin versus selective post-procedural rectal indometacin to prevent pancreatitis in patients undergoing endoscopic retrograde cholangiopancreatography: a multicentre, single-blinded, randomised controlled trial. Lancet. 2016; 387(10035):2293-301.

95. Kenshil S, D'Souza P, Sultanian P, Sandha GS, Bain V, Hundal R, et al. A prospective randomized clinical trial on the timing of rectal indomethacin administration for the prevention of post-ERCP pancreatitis: A preliminary report. Gastrointest Endosc. 2016;83(5):AB246. Abstract

96. Knol JA, Inman MG, Strodel WE, Eckhauser FE. Pancreatic response to crystalloid resuscitation in experimental pancreatitis. J Surg Res. 1987;43(5):387-92

97. Cuthbertson CM, Christophi C. Disturbances of the microcirculation in acute pancreatitis. Br J Surg. 2006;93(5):518-30

98. Bhoomagoud M, Jung T, Atladottir J, Kolodecik TR, Shugrue C, Chaudhuri $\mathrm{A}$, et al. Reducing extracellular $\mathrm{pH}$ sensitizes the acinar cell to secretagogue-induced pancreatitis responses in rats. Gastroenterology. 2009;137(3):1083-92.

99. Wu BU, Hwang JQ, Gardner TH, Repas K, Delee R, Yu S, et al. Lactated Ringer's solution reduces systemic inflammation compared with saline in patients with acute pancreatitis. Clin Gastroenterol Hepatol. 2011;9(8):710-7. e1.

100. de-Madaria E, Herrera-Marante I, Gonzalez-Camacho V, Bonjoch L, Quesada-Vázquez N, Almenta-Saavedra I, et al. Fluid resuscitation with lactated Ringer's solution vs. normal saline in acute pancreatitis: A triple-blind, randomized, controlled trial. United European Gastroenterol J. 2018;6(1):63-72.

101. Hoque R, Faroog A, Ghani A, Gorelick F, Mehal WZ. Lactate reduces liver and pancreatic injury in Toll-like receptor- and inflammasome-mediated inflammation via GPR81-mediated suppression of innate immunity. Gastroenterology. 2014;146(7):1763-74.

102. Radadiya D, Devani K, Arora S, Charilaou P, Brahmbhatt B, Young M, et al. Peri-procedural aggressive hydration for post endoscopic retrograde 
cholangiopancreatography (ERCP) pancreatitis prophylaxis: Meta-analysis of randomized controlled trials. Pancreatology. 2019;19(6):819-27.

103. Wu D, Wan J, Xia L, Chen J, Zhu Y, Lu N. The efficiency of aggressive hydration with lactated Ringer solution for the prevention of post-ERCP pancreatitis: A systematic review and meta-analysis. J Clin Gastroenterol. 2017;51(8):e68-e76.

104. Zhang ZF, Duan ZJ, Wang LX, Zhao G, Deng WG. Aggressive hydration with lactated Ringer solution in prevention of postendoscopic retrograde cholangiopancreatography pancreatitis: A meta-analysis of randomized controlled trials. J Clin Gastroenterol. 2017;51(3):e17-e26.

105. DiMagno MJ, Wamsteker EJ, Maratt J, Rivera MA, Spaete JP, Ballard DD, et al. Do larger periprocedural fluid volumes reduce the severity of post-endoscopic retrograde cholangiopancreatography pancreatitis? Pancreas. 2014;43(4):642-7.

106. Park CH, Paik WH, Park ET, Shim CS, Lee TY, Kang C, et al. Aggressive intravenous hydration with lactated Ringer's solution for prevention of post-ERCP pancreatitis: a prospective randomized multicenter clinical trial. Endoscopy. 2018;50(4):378-85.

107. Buxbaum J, Yan A, Yeh K, Lane C, Nguyen N, Laine L. Aggressive hydration with lactated Ringer's solution reduces pancreatitis after endoscopic retrograde cholangiopancreatography. Clin Gastroenterol Hepatol. 2014;12(2):303-7. e1.

108. Mok SRS, Ho HC, Shah P, Patel M, Gaughan JP, Elfant AB. Lactated Ringer's solution in combination with rectal indomethacin for prevention of post-ERCP pancreatitis and readmission: a prospective randomized, double-blinded, placebo-controlled trial. Gastrointest Endosc. 2017; 85(5):1005-13.

109. Hosseini $M$, Shalchiantabrizi $P$, Yektaroudy K, Dadgarmoghaddam M Salari M. Prophylactic effect of rectal indomethacin administration, with and without intravenous hydration, on development of endoscopic retrograde cholangiopancreatography pancreatitis episodes: $A$ randomized clinical trial. Arch Iran Med. 2016;19(8):538-43.

110. Hajalikhani M, Emami MH, Khodadoostan M, Shavakhi A, Rezaei M, Soluki R. Combination of diclofenac and aggressive hydration for the prevention of post-ERCP pancreatitis. Gastroenterol Hepatol Bed Bench. 2018;11(4):319-24

111. Luman W, Pryde A, Heading RC, Palmer KR. Topical glyceryl trinitrate relaxes the sphincter of Oddi. Gut. 1997;40(4):541-3.

112. Ding J, Jin X, Pan Y, Liu S, Li Y. Glyceryl trinitrate for prevention of post-ERCP pancreatitis and improve the rate of cannulation: a meta-analysis of prospective, randomized, controlled trials. PLoS One. 2013;8(10):e75645.

113. Sotoudehmanesh R, Eloubeidi MA, Asgari AA, Farsinejad M, Khatibian $\mathrm{M}$. A randomized trial of rectal indomethacin and sublingual nitrates to prevent post-ERCP pancreatitis. Am J Gastroenterol. 2014;109(6): 903-9.

114. Tomoda $\mathrm{T}$, Kato $\mathrm{H}$, Ueki $\mathrm{T}$, Akimoto $\mathrm{Y}$, Hata $\mathrm{H}$, Fujii $\mathrm{M}$, et al Combination of diclofenac and sublingual nitrates is superior to diclofenac alone in preventing pancreatitis after endoscopic retrograde cholangiopancreatography. Gastroenterology. 2019;156(6):1753-60. e1.

115. Luo H, Wang X, Zhang R, Liang S, Kang X, Zhang X, et al. Rectal indomethacin and spraying of duodenal papilla with epinephrine increases risk of pancreatitis following endoscopic retrograde cholangiopancreatography. Clin Gastroenterol Hepatol. 2019;17(8):1597-606. e5.

116. Aziz M, Ghanim M, Sheikh T, Sharma S, Ghazaleh S, Fatima R, et al Rectal indomethacin with topical epinephrine versus indomethacin alone for preventing Post-ERCP pancreatitis - A systematic review and meta-analysis. Pancreatology. 2020;20(3):356-61. 